\title{
Study of Residual Stress Compensation in Continuous Membrane Micromirrors Based on Surface Micromachining Processes
}

\author{
Qiang Wang ${ }^{1,2}$, Weimin Wang ${ }^{3,4} \oplus$, Liang Fang ${ }^{1}$, Chongxi Zhou ${ }^{1}$ and Bin Fan ${ }^{1, *}$ \\ 1 State Key Laboratory of Optical Technologies on Nano-Fabrication and Micro-Engineering, Institute of Optics \\ and Electronics, Chinese Academy of Sciences, Chengdu 610209, China; battery007@163.com (Q.W.); \\ fangl@ioe.ac.cn (L.F.); cxzhou@ioe.ac.cn (C.Z.) \\ 2 University of Chinese Academy of Sciences, Beijing 100049, China \\ 3 Key Laboratory of Optoelectronic Technology and Systems, Ministry of Education, Defense Key Disciplines \\ Laboratory of Novel Micro-Nano Devices and System Technology, College of Optoelectronic Engineering, \\ Chongqing University, Chongqing 400044, China; wwm@cqu.edu.cn \\ 4 Guangxi Key Laboratory of Automatic Detecting Technology and Instruments, Guilin University of \\ Electronic Technology, Guilin 541004, China \\ * Correspondence: fanbin@ioe.ac.cn; Tel.: +86-028-85100608
}

Citation: Wang, Q.; Wang, W.; Fang, L.; Zhou, C.; Fan, B. Study of Residual Stress Compensation in Continuous Membrane Micromirrors Based on Surface Micromachining Processes. Coatings 2021, 11, 289. https://doi. org/10.3390/coatings11030289

Academic Editor:

Alexander Tolstoguzov

Received: 1 February 2021

Accepted: 26 February 2021

Published: 3 March 2021

Publisher's Note: MDPI stays neutral with regard to jurisdictional claims in published maps and institutional affiliations.

Copyright: (c) 2021 by the authors. Licensee MDPI, Basel, Switzerland. This article is an open access article distributed under the terms and conditions of the Creative Commons Attribution (CC BY) license (https:// creativecommons.org/licenses/by/ $4.0 /)$.

\begin{abstract}
Residual stress is one of the key factors that directly determines the optical quality of micro-optical devices. With the same residual stress, the larger the aperture is, the worse the optical quality is. Therefore, continuous micromirrors are more affected by residual stress than segmented micromirrors. However, due to the complexity of boundary conditions, the influence of residual stress in segmented micromirror arrays on the device performance has been widely investigated in theory and practical applications, but only a few research results about the influence of residual stress in the continuous micromirror arrays have been reported. In this work, the residual stress both in continuous and segmented micromirror arrays is analyzed and summarized, then an accurate model for continuous micromirrors is developed. Compared with the existing models, it combines two additional factors, layer plate and point supported boundary conditions. Based on the proposed model, the change of critical stress of continuous micromirrors induced by different thicknesses of residual stress compensated membrane is theoretically investigated. Finally, the compensating experiment has been carried out, and the results show that the optical quality of micromirror can be remarkably improved, almost two orders of magnitude, with the introduction of residual stress compensation.
\end{abstract}

Keywords: residual stress; micromirror arrays; optical quality; stress compensation

\section{Introduction}

Surface micromachining is an important micro-electro-mechanical systems (MEMS) technique that has been used in various fields such as optical-phased arrays (OPAs) [1], digital micromirror devices (DMD) [2,3], grating light valves (GLV) [4], etc. However, the conventional thin membrane deposition method used in surface micromachining, such as low-pressure chemical vapor deposition (LPCVD) [5] and magnetron sputtering [6], would lead to residual stress in deposited thin membranes, deteriorating the mechanical and optical performance of the devices accordingly [7]. First of all, the residual stress will change the elastic coefficient of fabricated thin membranes and affect the load-deflection curve, causing impaired influence to the mechanical properties. These adverse influences on the mechanical properties can be amended during the design process with accurate models [8]. On the other hand, the optical factors such as curvature and surface flatness will be lowered by the deformation of the thin membranes induced by the residual stress, 
which would cause extreme detrimental effects on the performance of micro-opto-electromechanical systems (MOEMS) devices [9].

Micromirror arrays, which widely proved to be critically important MOEMS devices, can be classified as segmented micromirror arrays and continuous micromirror arrays according to the mirror boundary conditions [10-13]. The optical performance of segmented micromirror arrays due to the residual stress has been investigated by many researchers. Since its boundaries are totally or mostly free, the residual compressive stress or residual tensile stress would not cause any deformation of the mirror. None but the residual stress gradient can be responsible for the curvature of the mirror. Both the value of residual stress gradient and the flexural rigidity of the mirror decide the amplitude and shape of the mirror deformation [14].

According to the analysis, various methods have been proposed to solve these problems. One of the methods is to use zero stress materials, such as the single crystalline silicon substrate of bulk micromachining [15] or the single crystalline silicon layer of SOI technology $[16,17]$. This method can essentially solve the problem, but it is only available for specific processes and devices. Another method is to decrease the influence of residual stress instead of changing the fundamental fabricating process or materials such as increasing the thickness of the membrane to resist the curve [18], post processing (including using ion bombing to get rid of the tensile stress [19] and laser polishing [20]), and depositing an opposite-stressed compensation layer to decrease the whole residual stress gradient [21], etc. These methods could decrease the mirror curvature and enhance the optical performance of devices to a certain extent. Furthermore, due to the fact that the membrane would only be translated or tilted rather than deformed when it is driven, the static properties of the membrane such as steering angle, driven voltage etc., would not be affected by the changing of the thickness and the stress gradient distribution. However, all the proposed solutions aforementioned are only feasible for the segmented micromirrors, and there is still lack of a detailed theoretical analysis and experimental verifications for the continuous membrane micromirrors.

Continuous membrane micromirror arrays, which can also be called microdeformable mirrors, use localized discrete actuators to realize continuous local deformation in a whole membrane mirror [22]. These kinds of micromirror arrays are broadly applied to correct the wave front distortion and improve the imaging quality of telescopes, microscopes, free space optical communications, and so on [23]. The curvature mechanics of the continuous membrane micromirrors induced by residual stress and the generated problems when it is compensated for are different from the previous segmented micromirrors. In this work, we provide a detailed investigation of residual stress influence to the microdeformable mirrors theoretically and experimentally. The results obtained are essential for future development and quality control of micromirror arrays.

\section{Analysis and Modeling}

\subsection{Stress Compensation Theory for Continuous Micromirror Arrays}

For the case of continuous micromirror arrays, due to their fixed-fixed boundaries, the residual tensile stress in a mirror surface will guarantee the flatness of the membrane. On the contrary, the membrane would buckle if a residual compressive stress beyond the Euler buckling limit is present, as shown in Figure 1.
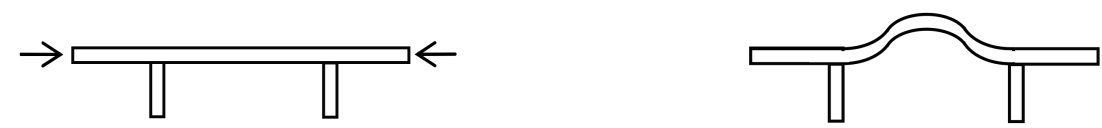

Figure 1. Deformation of continuous micromirrors induced by compressive stress.

The Euler buckling limit of a fixed-fixed beam is expressed as [8]

$$
\sigma_{\text {Euler }}=\frac{-\pi^{2}}{3} \frac{E H^{2}}{L^{2}}
$$


where $E, H$, and $L$ are the Young's module, thickness of the beam, and length of the beam respectively. According to Equation (1), the buckling of a membrane could be avoided if the total residual stress is shown to be tensile stress or compressive stress of which the intensity is less than the Euler buckling limit. Two methods can be applied to avoid the mirror surface deformation, one is increasing the thickness of mirror to increase the Euler buckling limit, and the other is redepositing a layer of membrane with a certain level of residual stress to realize the stress compensation. By adding another layer of stressed membrane, it can increase the thickness of the mirror and further increase the Euler buckling limit. On the other hand, the total residual stress of a deposited multilayer membrane can be deduced to the extent in which it is less than Euler buckling limit. No accurate membrane thickness and magnitude of residual stress is needed to avoid the mirror surface deformation for both methods aforementioned. The absolute smooth surface can be obtained as long as the total residual stress is less than Euler buckling limit.

For continuous mirrors, with the driving of an actuator, the supported pillar pushes the mirror surface to move up and down, inevitably leading to the deformation of the neighboring mirror surface. This deformation will be affected by the change of the mirror surface thickness, resulting from stress compensation. Also, due to the force transmission by mirror surface and pillar, the neighbor actuator would deform as well when an actuator is driven. An important parameter, actuator coupling, is introduced, which is defined by the rate of deformation degree between the actuated actuator and its nearest-neighbor unactuated actuator. Therefore, both of the driven voltages and actuator couplings will be influenced by the stress compensation. It is essential to take into account these two factors, driven voltage and actuator coupling, during the predesign process of devices. In addition, the mass of the mirror will increase by adding the thickness of the mirror layer, leading to the change of some dynamic characteristics such as the natural frequency of the mirror.

To sum up, there exist two differences in stress compensation between continuous and segmented micromirrors. One difference is the cause of the surface deformation. The deformation of segmented mirrors is induced by the gradient of residual stress, while a residual stress exceeding the Euler buckling limit is the cause of the deformation of continuous mirrors. The other difference is the influence of stress compensation methods. The performance of segmented mirrors will not be affected, but the fundamental performance parameters such as voltage and frequency of continuous mirrors will alter.

\subsection{Structural Model of Continuous Micromirror Array}

\subsubsection{Multilayer Sheets}

After the deposition of stress compensation membrane, the mirror surface changes to a multilayer structure instead of a single layer structure. The general single layer sheet model is not sufficient enough to describe the mechanical properties of multilayer structures. A new model is needed to analyze the mechanical features of the surface. Generally, as for isotropic multilayer sheets, if the Poisson's ratio of each layer is approximately identical, the multilayer structure can be regarded as a single layer structure with an equivalent bending rigidity $D^{*}$. We assume the mirror surface has $n$ layers of membranes, and assign the top layer as the first layer and the bottom layer as the $n_{t h}$ layer, and the top surface of the 1st layer as the zero point of $z$ axis coordinates, $E_{k}, v_{k}, t_{k-1}$, and $t_{k}$ are the Young's module, Poisson's ratio, height of upper surface, and height of lower surface of the $k$-th layer respectively. The equivalent bending rigidity $D^{*}$ can be calculated through Equation (2), where $D_{i}$ and $D_{v i}$ can be obtained through Equations (3) and (4) [24], where $i$ is changing from 1 to 3 .

$$
\begin{gathered}
D *=D_{3}-D_{2} \frac{D_{1} D_{2}-D_{v 1} D_{v 2}}{D_{1}^{2}-D_{v 1}^{2}} \\
D_{i}=\sum_{k=1}^{n} \frac{E_{k}}{1-v_{k}^{2}} \frac{t_{k}^{i}-t_{k-1}^{i}}{i}
\end{gathered}
$$




$$
D_{v i}=\sum_{k=1}^{n} \frac{E_{k} v_{k}}{1-v_{k}^{2}} \frac{t_{k}^{i}-t_{k-1}^{i}}{i}
$$

\subsubsection{Total Residual Distribution Force}

In addition, each layer has the residual stress $\sigma$, where positive values mean tensile stress and negative values compressive stress. The algebraic sum of the total residual stress can be treated as the in-plane distribution force of the multilayer sheets, which can be obtained by Equation (5).

$$
N *=\sum_{k=1}^{n} \sigma_{k}\left(t_{k}-t_{k-1}\right)
$$

\subsubsection{The Support Type}

The whole mirror surface can be treated as a thin plate supported by many pillars which are arranged in a rectangular array. Considering that the cross section area of pillar is much smaller than the mirror surface, the pillar could be regarded as a point. Suppose a plate supported by rows of equidistant points, and a concentrated load is applied on the center of each panel as shown in Figure 2a, where the hollow rectangle and the solid circular dot represent the pillar and the concentrated load, respectively. Then the relationship of load and deflection can be expressed as Equation (6) [25].

$$
w=0.0116 \frac{F a^{2}}{D}
$$

where $F$ is the load and $w$ is the deflection. The direction of concentrated load applied on the neighbor panel is opposite, as shown in Figure 2b, where the circular dot and cross represent a load with opposite direction. Given that the structure is symmetrical, and the deflection in the dashed line is zero and it is not applied bending moment, the rectangle enclosed by dashed line can be regarded as a simply supported rectangle plate. The relationship of load and deflection can also be expressed by Equation (6), where $a$ is the length of the side of the rectangle [26].

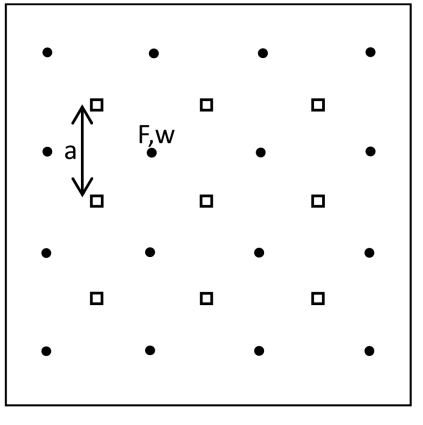

(a)

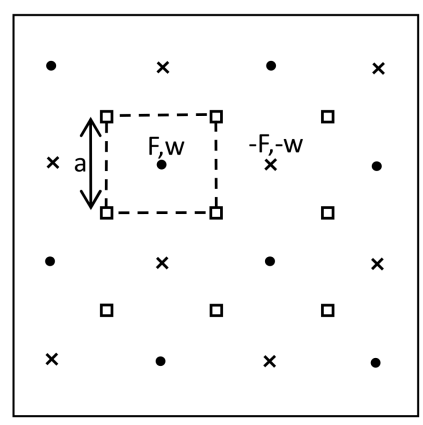

(b)

Figure 2. Schematic of a continuous micromirror array with a concentrated load. (a) Load in the middle of the plate. (b) Load in the middle of the adjacent plate is in the opposite direction.

Superimposing these two states, a complex applied load type can be obtained as shown in Figure 3a. Obviously, the relationship between deflection and load could be described by Equation (6) as well. It can be seen that the deflection value at the position where two opposite loads are applied is zero, similar to the pillar. 


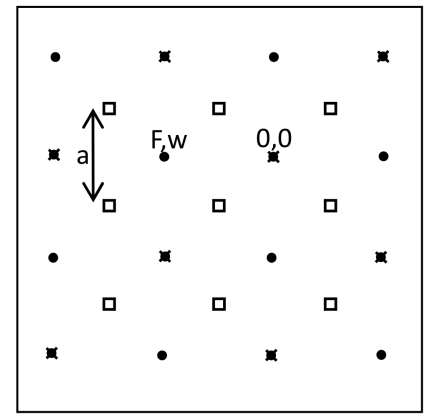

(a)

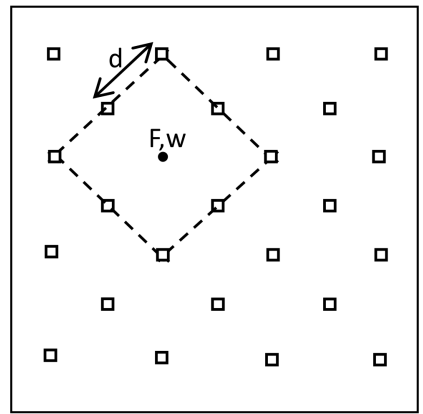

(b)

Figure 3. Schematic of a continuous micromirror array. (a) Superposing two kinds of concentrated load in the middle of the plate. (b) Concentrated load kind in practice.

For the practical continuum micromirror arrays, pillars arrange in both $x$ and $y$ directions with a periodicity of $d$, as shown in Figure $3 \mathrm{~b}$. When the pillar at the center of the rectangle enclosed by dashed line is pulled by actuator in the downward direction, it can be approximated as Figure 3a and now $a=\sqrt{2} \mathrm{~d}$. Based on Equation (6), the relationship of load and deflection can be expressed by Equation (7).

$$
w=0.0232 \frac{F d^{2}}{D}
$$

As for the case of square plate with all edges built in with a side length of $b$, when it has applied a central concentrated load $F$, the deflection of its center is [27]

$$
w=0.0056 \frac{F b^{2}}{D}
$$

It can be seen that the value of $w$ in Equations (7) and (8) is approximately the same when $b=2 d$, which means that the continuum mirror array with a pillar separation space of $d$ can be treated as an equivalent square plate with all edges fixed with a side length of $2 d$, as illustrated in Figure 4. Therefore, the analysis of continuous micromirror arrays could be substituted by the proposed model, i.e., a square plate with all edges fixed. Compared with this model, previously reported studies all treat the continuous micromirror array as a 2D structure, e.g., fixed-fixed beam [28].

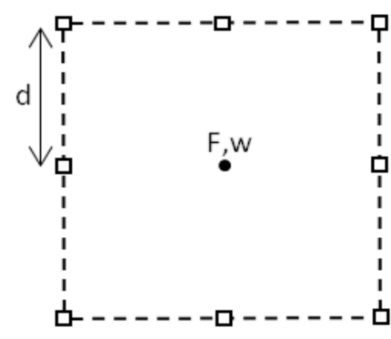

(a)

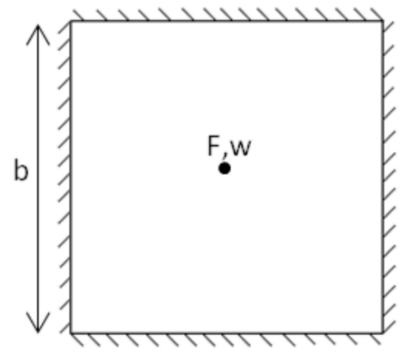

(b)

Figure 4. Model of continuous micromirror array with a concentrated load in the middle. (a) A square plate fixed by pillars. (b) A square plate clamped by peripheral edges.

\subsection{Euler Buckling Limit of Continuous Membrane Micromirror Array}

Equation (1) is the Euler buckling limit of a fixed-fixed beam. According to the analysis above, as for the point-supported continuous micromirror arrays with supported pillar distance of $a$, the square plate with all edges fixed model should be used to accurately describe the characteristic properties of mirror arrays. When we discuss the Euler buckling limit, only the residual stress is needed to be taken into account, and no external excitation is 
applied. So, the model can be regarded as a square plate with side length of $\sqrt{2} a$, as shown in Figure 5. Thus Euler critical stress $\sigma_{\text {Euler }}$ can be calculated through Equation (9) [29].

$$
\sigma_{\text {Euler }}=4.4 \frac{E t^{2}}{\left(1-v^{2}\right) b^{2}}
$$

where $E$ is Young's modulus, $t$ is thickness, and $v$ is the Poisson's ratio.

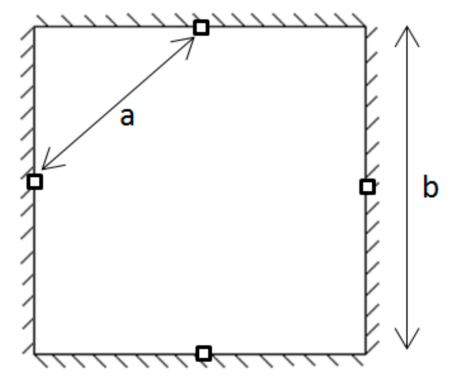

Figure 5. Schematic of the equivalent model when calculate the Euler stress.

Bending rigidity $D$ and uniform distributed force $N$ can be gotten through Equations (10) and (11) [30], such that the Euler critical limit distributed force $N_{\text {Euler }}$ can be calculated by Equation (12). Considering practical situations, the bending rigidity $D$ needs to be replaced by effective bending rigidity $D^{*}$, and further the Euler critical force of a certain layer can be obtained by Equation (5) and the known thickness and residual stress of each layer.

$$
\begin{array}{r}
D=\frac{1}{12}\left(\frac{E t^{3}}{1-v^{2}}\right) \\
N=\sigma t \\
N_{\text {Euler }}=\frac{-52.8 D}{b^{2}}
\end{array}
$$

In our study, polycrystalline silicon is used to prepare the mirror surface, and the key parameters such as Young's modulus, thickness, pillar distance, Poisson's ratio, residual stress and density are listed in Table 1.

Table 1. Key parameters of the mirror.

\begin{tabular}{cccccc}
\hline$E(\mathrm{GPa})$ & $\boldsymbol{t}(\mu \mathrm{m})$ & $\boldsymbol{a}(\mu \mathrm{m})$ & $v(-)$ & $\sigma(\mathrm{MPa})$ & $r\left(\mathrm{~kg} / \mathrm{m}^{3}\right)$ \\
\hline 160 & 1.5 & 410 & 0.226 & -8 & 2300 \\
\hline
\end{tabular}

Chromium ( $\mathrm{Cr}$ ) membrane which possesses large tensile stress is adopted as a stress compensation layer, and the material parameters of $\mathrm{Cr}$ film are listed in Table 2.

Table 2. Key parameters of the $\mathrm{Cr}$ layer.

\begin{tabular}{cccc}
\hline$E(\mathrm{GPa})$ & $\boldsymbol{v}(-)$ & $\sigma(\mathrm{MPa})$ & $r\left(\mathbf{k g} / \mathbf{m}^{3}\right)$ \\
\hline 140 & 0.3 & 1500 & 7190 \\
\hline
\end{tabular}

Substituting the parameters listed in Tables 1 and 2 into equations above, a curve of Euler critical stress of mirror layer vs. Cr thickness is obtained, as depicted in Figure 6. 


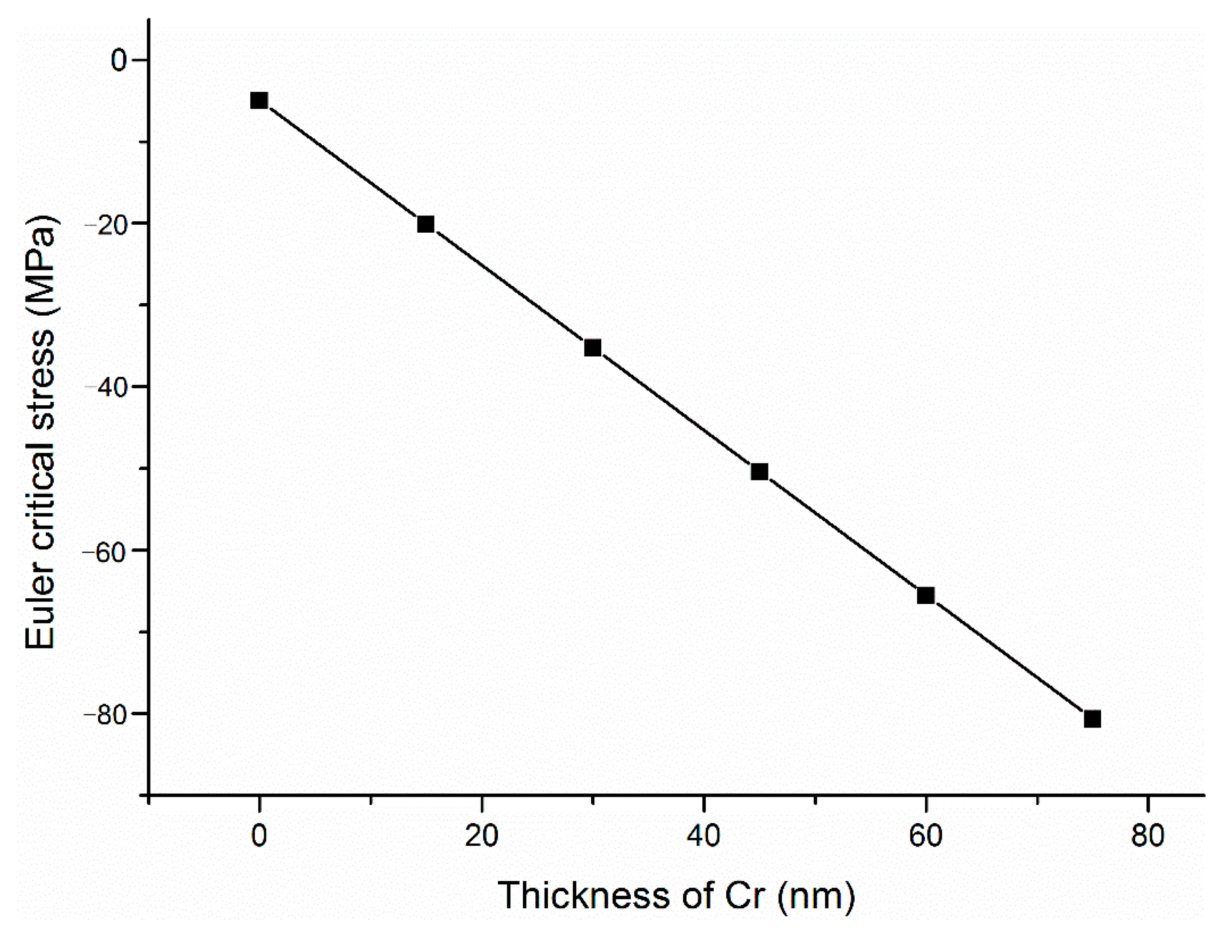

Figure 6. Relationship between Euler stress and Cr thickness.

With the enhancement of $\mathrm{Cr}$ membrane thickness, the Euler critical stress of the mirror layer increase dramatically. As Figure 6 illustrates, the Euler critical stress of the mirror surface is about $-5 \mathrm{MPa}$ without $\mathrm{Cr}$. In contrast, the residual stress of polycrystalline thin membrane fabricated by the general surface micromachining technique is much larger, very easily leading to mirror surface buckling. On the other hand, when the thickness of Cr membrane increases to $60 \mathrm{~nm}$, even when the polycrystalline silicon residual stress is as large as $-60 \mathrm{MPa}$, the mirror surface can still be kept smooth because the resultant residual stress is less than the critical value.

\section{Experimental Results and Discussion}

The mirror array is fabricated by a commercial surface micromachining process PolyMUMPs supplied by MEMSCAP Co. (Durham, NC, USA) [31]. This process consists of a nitride isolation layer, two oxide sacrificial layers, three polysilicon layers, and a metal layer (gold). The schematic diagram of a partial fabricated continuous mirror array is depicted in Figure 7. Only 9 unit cells are shown in the diagram, and the surface is connected to the actuator through the supported pillars.

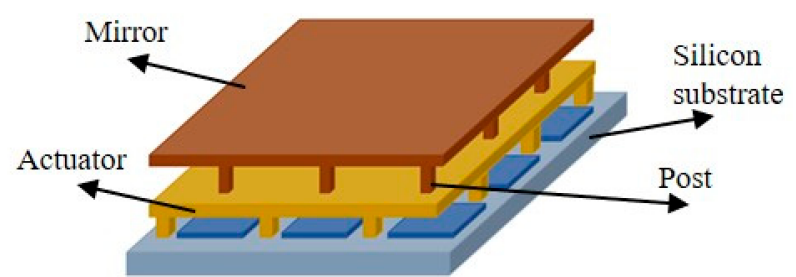

Figure 7. Schematic diagram of the partial continuous micromirror array.

The residual stress of fabricated polycrystalline silicon of PolyMUMPs is $-8 \mathrm{MPa}$, which exceeds the Euler critical stress as aforementioned. Consequently, the mirror buckling happens inevitably after finishing the fabrication. In order to describe the surface deformation, including surface figure and roughness, Peak-to-Valley (PV) and Root Mean Square (RMS) are utilized. PV is the absolute value between the highest and lowest peaks, and RMS is the root-mean-square parameter corresponding to roughness Ra. Optical 
profilometers are one of the most widely used pieces of equipment in measuring surface deformation [32]. Figure 8a shows the interference micrograph of the surface of the micromirror array, which is achieved by a Zygo optical profiler [33]. The denser the light and dark interference fringes are, the larger the surface deformation is. Figure $8 b$ is the profile of Figure $8 \mathrm{a}$, which is calculated from the interference pattern, according to a patent algorithm belonging to Zygo Corporation (Middlefield, OH, USA). PV and RMS, two statistics of Figure $8 \mathrm{~b}$, are as large as 9.83 and $2.71 \mu \mathrm{m}$, which is too large to be used practically.

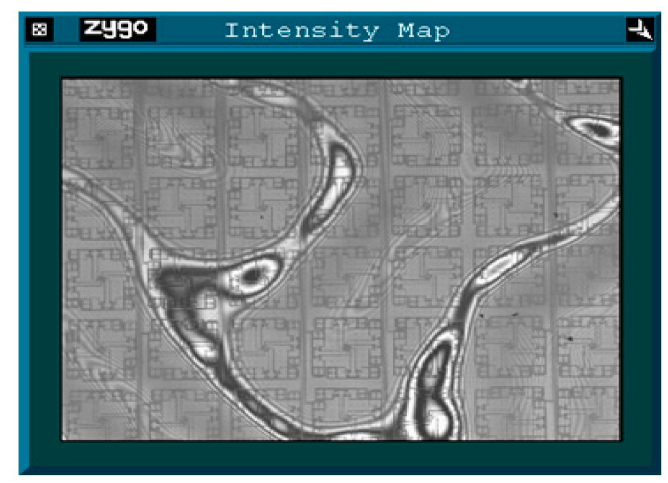

(a)

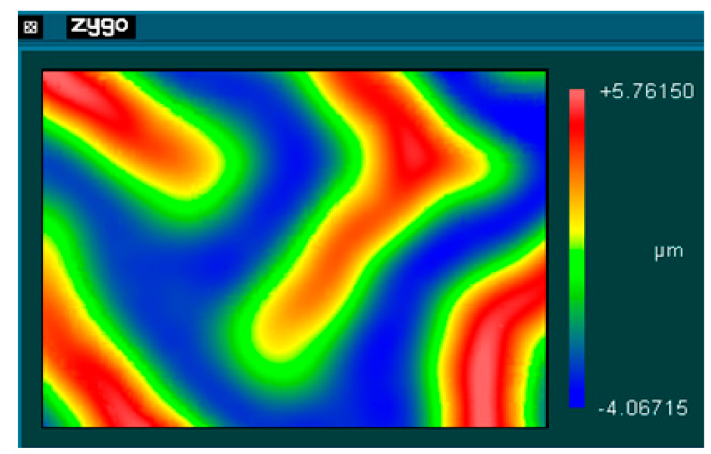

(b)

Figure 8. (a) Interference pattern of the fabricated mirror in Zygo profiler. (b) Surface profile measured by Zygo profiler.

In order to realize the stress compensation, the magnetron sputtering method is used to deposit a layer of $\mathrm{Cr}$ membrane with tensile stress onto the mirror surface. The residual stress intensity is measured through a cantilever structure. Table 3 shows the measured results for different thickness of $\mathrm{Cr}$ membrane, indicating that the stress values decrease slightly $(<10 \%)$ as $\mathrm{Cr}$ thickness increases by $50 \%$. For simplicity, this small variance is ignored in the forenamed theoretical analysis and the stress of $\mathrm{Cr}$ is chosen as $1500 \mathrm{MPa}$.

Table 3. Relation between Cr membrane thickness and stress intensity.

\begin{tabular}{cc}
\hline Thickness $\boldsymbol{t}(\mathbf{n m})$ & Residual Stress $\boldsymbol{\sigma} \mathbf{( M P a )}$ \\
\hline 40 & 1527 \\
50 & 1463 \\
60 & 1380 \\
\hline
\end{tabular}

Because the $\mathrm{Cr}$ membrane is sputtered after the release and dicing of the die, a photomask cannot be adopted to pattern the membrane. The shadow masking technique, as depicted in Figure 9, is employed. The shadow mask is suspended above the die and does not make contact with the surface of the die, to avoid damaging the die. There is a square hole in the middle of the mask, hence after sputtering, a Cr membrane of the same shape and size is left in the center of the micromirror. 


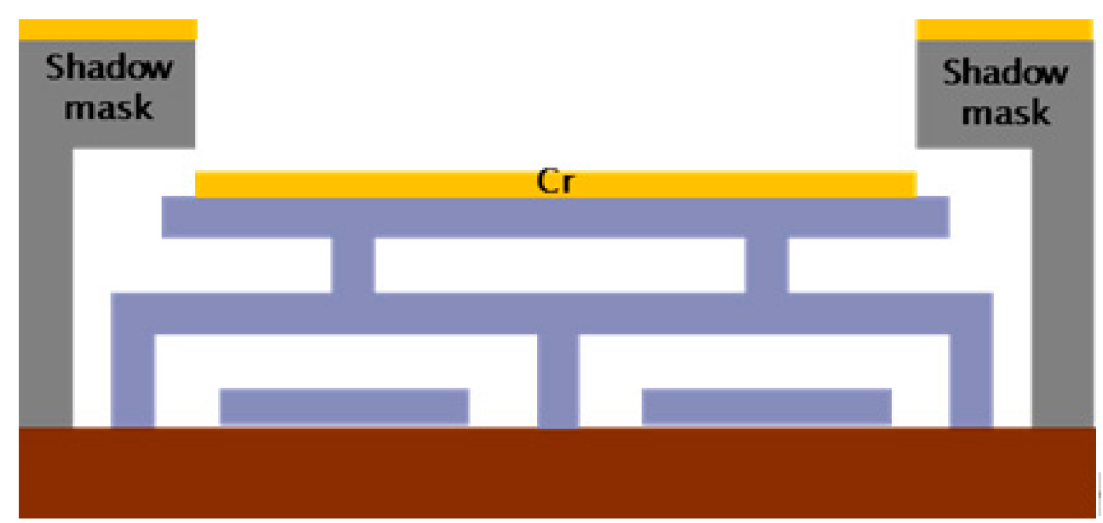

(a)

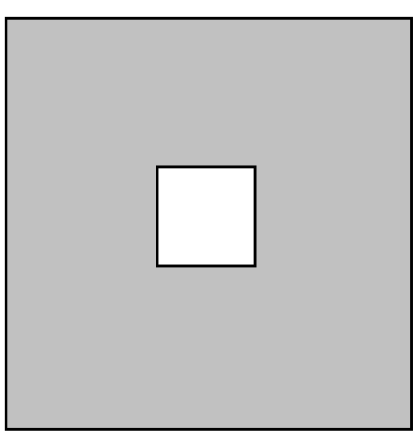

(b)

Figure 9. (a) A schematic of shadow masking technique. (b) Top view of the shadow mask.

We decide to use $40 \mathrm{~nm}$ thick deposited Cr membrane to get a better stress compensation result. In this case, the theoretical mirror surface Euler critical stress is about $-45 \mathrm{MPa}$, which is much higher than the actual residual stress of the mirror surface $(\sim-10 \mathrm{MPa})$. Hence, even if the residual stress is slightly larger due to the uniformity of the process, the compensation effect can be guaranteed. Figure 10a is the interference micrograph of the area shown in Figure 8a after being coated by $40 \mathrm{~nm} \mathrm{Cr}$, and illustrating an evident stress compensation effect through the sparse interference fringes. Figure $10 \mathrm{~b}$ is the corresponding measured result of the surface profile. The PV and RMS of the mirror surface decrease dramatically to be 0.39 and $51 \mathrm{~nm}$, which can satisfy the practical requirement initially.

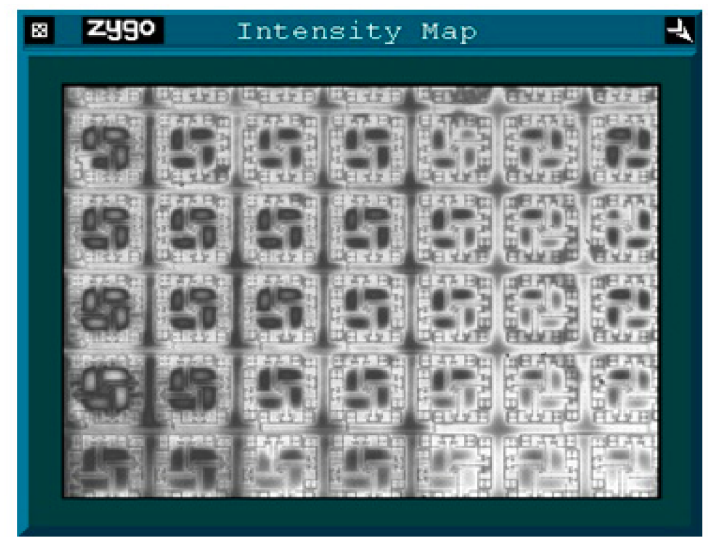

(a)

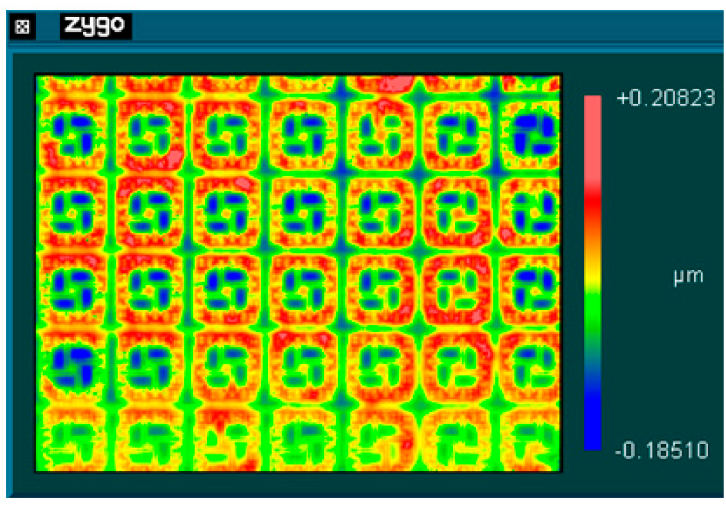

(b)

Figure 10. Schematic diagram of the micromirror after depositing $40 \mathrm{~nm} \mathrm{Cr}$ membrane. (a) Interference figure. (b) Profile plot. 


\section{Conclusions}

In this work, the residual stress compensation in continuous micromirror arrays is investigated. The buckling effect generated by residual stress and the stress compensation difference between continuous and segmented micromirror surfaces are analyzed. An equivalent physical model is formulated by taking into account support method and multilayer structure factors. The critical stress is investigated by the proposed model. Experimental measurement is done to verify the theoretical analysis, and the results show that the PV value decreases from 10 to $0.5 \mu \mathrm{m}$, RMS from $3 \mu \mathrm{m}$ to $50 \mathrm{~nm}$.

Author Contributions: Writing—original draft preparation, Q.W. and W.W.; theoretical derivation, W.W.; fabrication, Q.W.; measurement, Q.W. and W.W.; supervision, B.F.; project administration, C.Z.; funding acquisition, L.F. All authors have read and agreed to the published version of the manuscript.

Funding: This research was supported by the National Key Research and Development Program of China (Grant Nos. 2019YFA0706001 and 2016YFB0500200), Guangxi Key Laboratory of Automatic Detecting Technology and Instruments (Grant No. YQ18201), and CAS “Light of West China" Program.

Institutional Review Board Statement: Not applicable.

Informed Consent Statement: Not applicable.

Data Availability Statement: The data that support the findings of this study are available from the corresponding author upon reasonable request.

Conflicts of Interest: The authors declare no conflict of interest.

\section{References}

1. Wang, Y.; Zhou, G.; Zhang, X.; Kwon, K.; Blanche, P.-A.; Triesault, N.; Yu, K.-S.; Wu, M.C. 2D broadband beamsteering with large-scale mems optical phased array. Optica 2019, 6, 557-562. [CrossRef]

2. Hornbeck, L.J. Current status of the digital micromirror device (DMD) for project television applications. In Proceedings of IEEE International Electron Devices Meeting, Washington, DC, USA, 5-8 December 1993; pp. 381-384.

3. Smith, B.; Hellman, B.; Gin, A.; Espinoza, A.; Takashima, Y. Single chip lidar with discrete beam steering by digital micromirror device. Opt. Express 2017, 25, 14732-14745. [CrossRef] [PubMed]

4. Ashida, Y.; Hamann, S.; Landry, J.; Solgaard, O. Conjugated MEMS phased arrays for large field of view random access scanning. IEEE Photonics Technol. Lett. 2020, 32, 1291-1294. [CrossRef]

5. Yu, H.; Chen, H.; Li, J.; Wang, C. An in situ growth method for property control of lpcvd polysilicon film. Chin. Opt. Lett. 2004, 2, 489-492.

6. Chason, E.; Guduru, P.R. Tutorial: Understanding residual stress in polycrystalline thin films through real-time measurements and physical models. J. Appl. Phys. 2016, 119, 191101. [CrossRef]

7. Ryan, P.J.; Cornelissen, S.A.; Lam, C.V.; Bierden, P.A. In Performance analysis of two high actuator count MEMS deformable mirrors. In SPIE Proceedings, MEMS Adaptive Optics VII; Olivier, S.S., Bifano, T.G., Kubby, J., Eds.; SPIE: Bellingham, WA, USA, 2013.

8. Senturia, S.D. Microsystem Design; Kluwer Academic: New York, NY, USA, 2001; pp. 136-162.

9. Cowan, W.D.; Bright, V.M.; Lee, M.K.; Comtois, J.H.; Michalicek, M.A. Design and Testing of Polysilicon Surface-Micromachined Piston Mirror Arrays. In SPIE Proceedings, Spatial Light Modulators; SPIE: Bellingham, WA, USA, 1998; pp. 60-70.

10. Chen, J.; Ma, J.; Mao, Y.; Liu, Y.; Li, B.; Chu, J. Experimental evaluation of a positive-voltage-driven unimorph deformable mirror for astronomical applications. Opt. Eng. 2015, 54, 117103. [CrossRef]

11. Zamkotsian, F.; Lanzoni, P.; Barette, R.; Helmbrecht, M.; Marchis, F.; Teichman, A. Cryogenic testing of moems deformable mirror for future optical instrumentation. In Proceedings of the International Conference on Optical MEMS and Nanophotonics (OMN), Santa Fe, NM, USA, 13-17 August 2017; pp. 1-2.

12. Jung, I.W.; Peter, Y.-A.; Carr, E.; Wang, J.-S.; Solgaard, O. Single-crystal-silicon continuous membrane deformable mirror array for adaptive optics in space-based telescopes. IEEE J. Sel. Topics Quantum Electron. 2007, 13, 162-167. [CrossRef]

13. Jia, K.; Samuelson, S.R.; Xie, H. High-fill-factor micromirror array with hidden bimorph actuators and tip-tilt-piston capability. J. Microelectromech. Syst. 2011, 20, 573-582. [CrossRef]

14. Liu, C. Foundations of MEMS, 2nd ed.; China Machine Press: Beijing, China, 2006; pp. 62-64.

15. Strathman, M.; Liu, Y.; Li, X.; Lin, L.Y. Dynamic focus-tracking mems scanning micromirror with low actuation voltages for endoscopic imaging. Opt. Express 2013, 21, 23934-23941. [CrossRef] [PubMed]

16. Su, G.D.; Toshiyoshi, H.; Wu, M.C. Surface micromachined 2D optical scanners with high performance single-crystal silicon micromirrors. Photonics Technol. Lett. 2001, 13, 606-608. [CrossRef] 
17. Liu, X.; Takahashi, T.; Konishi, M.; Motohara, K.; Toshiyoshi, H. Random access addressing of mems electrostatic shutter array for multi-object astronomical spectroscopy. Micromachines 2020, 11, 782. [CrossRef] [PubMed]

18. Dagel, D.J.; Cowan, W.D.; Spahn, O.B.; Grossetete, G.D.; Grine, A.J.; Shaw, M.J.; Resnick, P.J.; Bernhard Jokiel, J. Large-stroke mems deformable mirrors for adaptive optics. J. Microelectromechanical Syst. 2006, 15, 572-583. [CrossRef]

19. Bifano, T.G.; Johnson, H.T.; Bierdon, P.; Mali, R.K. Elimination of stress-induced curvature in thin-film structures. J. Microelectromech. Syst. 2002, 11, 592-597. [CrossRef]

20. Ukar, E.; Lamikiz, A.; Martínez, S.; Tabernero, I.; Lopez de Lacalle, L.N. Roughness prediction on laser polished surfaces. J. Mater. Process. Technol. 2012, 212, 1305-1313. [CrossRef]

21. Cao, K.; Liu, W.; Talghader, J.J. Curvature compensation in micromirrors with high-reflectivity optical coatings. J. Microelectromech. Syst. 2001, 10, 409-417. [CrossRef]

22. Fernández, B.R.; Bouchti, M.A.; Kubby, J. High-stroke, high-order mems deformable mirrors. J. Micro Nanolithography MEMS MOEMS 2013, 12, 033012. [CrossRef]

23. Bifano, T.G.; Perreault, J.; Mali, R.K.; Horenstein, M.N. Microelectromechanical deformable mirrors. J. Sel. Topics Quantum Electron. 1999, 5, 83-86. [CrossRef]

24. Pister, K.S.; Dong, S.B. Elastic bending of layered plates. J. Eng. Mech. Div. Proc. Am. Soc. Civ. Eng. 1959, 85, 1-10.

25. Pozzati, P. Contributo al calcolo dei solai a fungo. Riv. Math. Univ. Parma 1951, 2, 123-131.

26. Young, W.C.; Budynas, R.G. Roark's Formulas for Stress and Strain; McGraw-Hill: New York, NY, USA, 2002 ; pp. $502-547$.

27. Timoshenko, S.; Woinowsky-Krieger, S. Theory of Plates and Shells; McGraw-Hill: New York, NY, USA, 1959.

28. Hardy, J.W. Adaptive Optics for Astronomical Telescopes; Oxford University Press: New York, NY, USA, 1998.

29. Timoshenko, S.P.; Gere, J.M. Theroy of Elastic Stability; Science Press: Beijng, China, 1965; pp. 410-413.

30. Wang, Z. Microsystem Design and Fabrication; Tsinghua Press: Beijing, China, 2007.

31. Cowen, A.; Hardy, B.; Mahadevan, R.; Wilcenski, S. Polymumps Design Handbook, Revision 13.0; MEMSCAP Inc.: Durham, NC, USA, 2011.

32. Rodríguez-Barrero, S.; Fernández-Larrinoa, J.; Azkona, I.; Lopez de Lacalle, L.N.; Polvorosa, R. Enhanced performance of nanostructured coatings for drilling by droplet elimination. Mater. Manuf. Process. 2016, 31, 593-602. [CrossRef]

33. Grigg, D.; Felkel, E.; Roth, J.; Lega, X.C.D.; Deck, L.; Groot, P.D. Static and dynamic characterization of MEMS and MOEMS devices using optical interference microscopy. In SPIE Proceedings, MEMS, MOEMS and Micromachining; SPIE: Bellingham, WA, USA, 2004; pp. 55-61. 